\title{
EL DOLOR IRRUPTIVO ONCOLÓGICO O LA CENICIENTA REVELADA
}

\author{
ONCOLOGIC BREAKTHROUGH PAIN OR THE UNCOVERED CINDERELLA
}

\author{
Yolanda Escobar Álvarez', Dulce Rodríguez Mesa², Jordi Moya Riera ${ }^{3}$ y Maite Murillo \\ Gonzalez ${ }^{4}$ \\ 1 Servicio de Oncología Médica. Hospital General Universitario Gregorio Marañón, Madrid \\ 2 Unidad de Cuidados Paliativos, Hospital Sant Joan de Reus, Tarragona \\ 3 Unidad del Dolor, Hospital Mateu Orfila, Menorca \\ ${ }^{4}$ Servicio de Oncología Radioterápica, Hospital 12 de Octubre, Madrid
}

Resumen

Al igual que la Cenicienta es un personaje de cuento de hadas que representa el arquetipo de persona joven que consigue la plenitud después de amargas pruebas debido a la incomprensión y falta de atención de su entorno familiar y social, el dolor irruptivo oncológico (DIO) es un problema clínico relevante por su frecuencia y su intensidad pero también víctima del desconocimiento y la falta de interés. A pesar de su alta prevalencia e intensidad, sigue siendo un síntoma mal conocido $y$, por ello, poco investigado e incorrectamente tratado. Sin embargo, son factibles su diagnóstico y evaluación correctos y el tratamiento ha evolucionado al aparecer fármacos basados en el fentanilo administrado a través de las mucosas que se ajustan, como el pie de Cenicienta al zapato de cristal, a las características del dolor irruptivo. Otros pies se habrán probado el zapatito y se habrán mutilado para intentar encajar en él pero sólo la misteriosa habitante de los fogones puede legitimar la propiedad y, por tanto, ser la elegida del príncipe.

Palabras clave: Dolor irruptivo, oncología, fentanilo.

\section{Abstract}

Cinderella is a fairy tale character who represents the archetypal young person getting happiness after bitter experiences due to misunderstanding and lack of attention from her family and social environment. Similarly, breakthrough pain is a relevant clinical problem due to its frequency and intensity but it is also a victim of lack of knowledge and lack of interest. Breakthrough pain, despite is a relevant clinical problem because of its high prevalence and its intensity, remains poorly understood and therefore improperly treated and poorly investigated. However, proper diagnosis and assessment are feasible, and treatment includes drugs as fentanyl administered through mucous membranes that fits to the characteristics of the breakthrough pain as the shoe of glass slipper fits in the foot of Cinderella. Other feet have been tested with no luck but only the mysterious kitchen's girl can legitimize the ownership and therefore be the chosen by the prince.

Key words: Breakthrough pain, oncology, fentayl.

\section{Correspondencia:}

Yolanda Escobar Álvarez

Servicio de Oncología Médica

Hospital General Universitario Gregorio Marañón

C/ Doctor Esquerdo, 4628007 Madrid

E-mail: yolandaesco@yahoo.es 


\section{INTRODUCCIÓN}

Hay personajes en los cuentos infantiles que concitan toda la lástima y la simpatía de los lectores porque se comprende que reciben de su entorno un trato injusto y carecen de valedores capaces de reivindicar sus verdaderas cualidades físicas y morales. Tal es el caso de La Cenicienta, doncella de extraordinaria belleza y bondad que vive oculta entre las cenizas del hogar de una casa en la que es menospreciada por personas incapaces de valorar sus aptitudes.

El dolor irruptivo oncológico (DIO) padece una situación semejante: su importancia y su intensidad pasan desapercibidas y son minusvaloradas en el amplio contexto de las manifestaciones del cáncer $y$, en concreto, del dolor crónico, verdadero protagonista de los desvelos profesionales de los oncólogos en el control de síntomas.

El objetivo de este artículo es sacarlo de la cocina y ponerlo donde se merece, en el salón de baile del palacio, para que pueda mostrarse y entenderse.

\section{CONCEPTOS Y DEFINICIÓN}

En este apartado ya empiezan las similitudes, pues tanto para La Cenicienta como para el DIO hay varias versiones, algunas bastante antiguas.

La Cenicienta es un personaje de cuento de hadas del que se conocen varias formas, tanto orales como escritas y con una nomenclatura diferente según los países. Las dos versiones más conocidas son la del francés Charles Perrault, de 1697 y la de los alemanes hermanos Grimm, de 1812. Ambas son parecidas pero con detalles diferentes que llegan a cambiar el sentido de los acontecimientos y a manifestar una personalidad distinta del mismo personaje ${ }^{(1,2)}$.

Igualmente, el DIO se ha concebido de diversos modos a lo largo del tiempo y, aunque su historia es mucho más reciente que la del personaje del cuento, las siguientes definiciones dan idea de que, en torno a un concepto común, pueden aparecer más o menos elementos definitorios. Veamos algunos ejemplos:

"El DIO es una exacerbación aguda del dolor de rápida aparición, corta duración e intensidad de moderada a elevada que sufre un paciente cuyo dolor basal está estabilizado y controlado"(3). "Es un incremento transitorio de dolor en un paciente con cáncer, que tiene el dolor basal estable y tratado con opioides"(4). "El dolor irruptivo es una exacerbación transitoria del dolor que se produce de forma persistente y estable con tratamiento"(5). "Es una exacerbación transitoria de dolor experimentado por un paciente con el dolor basal relativamente estable y adecuadamente controlado"(6). Parecidas pero diferentes, las definiciones apuntan a cierta confusión conceptual.

\section{ETIOLOGíA}

¿Cómo ha llegado La Cenicienta a su terrible situación?

Han sido varias las causas: en primer lugar, su madre ha muerto $y$, con ello, ha perdido a la persona que la hubiera protegido por encima de todo; por otro lado, su padre se ha vuelto a casar y ha centrado su atención en la nueva esposa, la cual aporta sus propias hijas. Resulta doloroso pensar en la insensibilidad de un padre que no hace nada por sacar a su hija de esa situación degradante pero ni siquiera queda claro que él conozca toda la verdad. Por último, la envidia y la rivalidad soterradas de unas hermanastras malvadas capitaneadas por su calculadora madre, sirven de acicate al deseo de ver humillada y afeada a la verdadera heredera y hermosa muchacha: se suprime una competidora y se gana una criada $^{(7)}$.

En el caso del DIO, el origen también es múltiple y no siempre perceptible ${ }^{(8)}$. Sa- 
bemos que la causa más frecuente es la propia enfermedad tumoral, mediante los procesos habituales de compresión, infiltración y otros; sin embargo, no debemos despreciar el papel del propio tratamiento antitumoral en su génesis, pues tanto la cirugía como la quimioterapia, radioterapia y terapias biológicas pueden desencadenarlo. Por último, hay otro factor causal, que está constituído por sucesos concomitantes al tumor, aunque no directamente relacionados con él, como puede ser un herpes zóster facilitado por la inmunodepresión y evolucionado a neuralgia postherpética.

\section{CARACTERÍSTICAS}

Si hay algo que define a La Cenicienta es la ceniza precisamente pues, a pesar de ser un miembro más de una familia pudiente, su madrastra y hermanastras la utilizan como servicio doméstico y la excluyen de toda la vida social que se teje con el objetivo de emparejar a las poco agraciadas hijas de la esposa actual del padre de familia, viudo de la madre de Cenicienta. Es decir, la muchacha carece de nombre y vive sucia y afeada, en la cocina, ocupada en las tareas más bajas, a pesar de ser la auténtica heredera. Ni siquiera es invitada al baile palaciego organizado para encontrar a la futura esposa del príncipe heredero del que un día será rey.

El DIO también ocupa una posición poco afortunada en la escala de importancia de los síntomas oncológicos; tanto es así que a la imprecisión conceptual se une la dificultad de saber cuál es su verdadera prevalencia en la clínica y su reparto real en los distintos tipos de tumores, donde la frecuencia del dolor crónico está mucho mejor determinado.

Está publicado que aparece en aproximadamente un $50 \%$ de los pacientes oncológicos, aunque los datos de prevalencia oscilan entre el 20 y el $95 \%{ }^{(9)}$.
Mejor conocidos son, en la actualidad, sus atributos: rapidez de instauración, con una media de 3 a 5 minutos, elevada intensidad, corta duración, con una media de 15 a 30 minutos y número de episodios diarios con media de 1 a $4^{(10)}$.

\section{CLASIFICACIÓN}

Cualquier intento de clasificación de los personajes de La Cenicienta pasa por una gran dicotomía: los buenos y los malos; entre los primeros está la propia Cenicienta, sus amigos los pájaros, los ratones, el árbol o el hada, según las versiones. Entre los malos, por supuesto, la madrastra y las hermanastras (aunque éstas no se comportan igual en la versión de Grimm que en la de Perrault). Quedan, sin embargo, personajes neutros como el padre de Cenicienta, cuyo comportamiento moral no llega a explicarse ni tampoco sus motivaciones (ciego de amor por su segunda mujer, demasiado ocupado con sus actividades como para conocer lo que ocurre en su casa o bien débil de carácter para oponerse al oprobio de su propia hija). En cuanto al príncipe y su padre el rey, está claro que desean una boda "democrática" basada en el enamoramiento del heredero, pues se supone que todas las doncellas del reino están más que encantadas de resultar elegidas pero no hay muchos datos para clasificarlos entre los buenos o entre los malos. La elección por parte del príncipe de la misteriosa joven que se ausenta antes de terminar la fiesta y pierde un zapato de cristal pone en marcha un mecanismo de indagaciones que revoluciona la vida doméstica de todo el reino.

Clasificar, por lo tanto, es siempre complicado, incompleto $y$, seguramente, sesgado, pero es una forma de poder encuadrar las cosas y los hechos antes de actuar sobre ellos.

Cuando nos referimos al DIO también necesitamos encuadrarlo en un esquema 
que facilite nuestra toma de decisiones. Sabemos que puede clasificarse según dos aspectos fundamentales: fisiopatología y factores desencadenantes ${ }^{(11)}$.

Las variantes fisiopatológicas del DIO son las mismas que las del dolor crónico oncológico, es decir: nociceptivo (subdividido en somático y visceral), neuropático y mixto (con componentes nociceptivos y neuropáticos).

Según los factores desencadenantes, se clasifica en:

- Idiopático, cuando dichos factores son desconocidos

- Incidental, cuando hay algún factor conocido implicado en su aparición, que puede ser voluntario o involuntario; dentro del tipo incidental podemos considerar el DIO procedimental, provocado por maniobras diagnósticas o terapéuticas.

El dolor por fallo de fin de dosis no se considera en la actualidad un DIO (12) sino que se relaciona con un mal control del dolor basal por fallo posológico o dosis insuficiente.

Esta clasificación es muy importante porque permite considerar la posibilidad de prevenir antes de su aparición aquellas formas de DIO que se asocien a actos voluntarios o a procedimientos, mientras que el idiopático y el que se asocia a actos involuntarios (fisiológicos o patológicos) sólo puede ser tratado una vez aparecido.

\section{DIAGNÓSTICO}

En el caso de La Cenicienta es evidente- como pudo constatar el príncipe- que conocerla es amarla pero ¡ay! ¡qué difícil resulta conocerla!. Es imposible distinguir debajo de la capa de polvo y ceniza la belleza de sus rasgos y tampoco puede evocarse su apostura y estilo entre los harapos cotidianos. Además, presentada como criada, ya condiciona la impresión de su visitante. Todo está en su contra y se pre- cisa alguna prueba que ayude a encontrar la verdad; esa prueba es el zapato perdido.

Todo el proceso de identificación comienza con el envío de un emisario a las casas de las jóvenes que fueron invitadas al baile de palacio, y esto ya introduce una primera selección. Una vez localizada cada candidata, viene la prueba fundamental: el pie debe ser del tamaño exacto del zapato, sin trampas: esa es la selección definitiva pues, por el tamaño minúsculo del zapato, muy pocas muchachas podrán calzarlo; cuando resulta que ninguna de las invitadas "oficiales" es la misteriosa candidata a princesa, hay que indagar más, hasta forzar la aparición de la habitante entre cenizas....que resulta ser, contra toda lógica, la verdadera dueña del zapato...y del pie.

En el caso del DIO, la atención también tiende a enfocarse sobre el dolor crónico, mejor conocido y más identificable. Para poder acceder al DIO es preciso diagnosticarlo correctamente ${ }^{(13)}$, empezando por una anamnesis específica que reproduzca las preguntas características de la evaluación del dolor para la situación particular del dolor irruptivo; se precisa conocer tanto los atributos como la cronología, la posible irradiación, los procedimientos de alivio o intensificación ${ }^{(14)} y$, en general, como en el cuento de Cenicienta, donde la clave es llegar a distinguirla de las hermanastras omnipresentes, hacer el diagnóstico diferencial con otras formas de dolor, para lo cual, en ausencia de un duque portador y probador del zapato, podamos usar otro instrumento útil, en este caso el algoritmo de Davies; se trata de un método sencillo que va descartando opciones, como la búsqueda de la dueña del zapatito: primero se sabe si el paciente padece dolor crónico o no; sólo en caso de que la respuesta sea afirmativa se procede a preguntar si está controlado con los analgésicos. Si la respuesta es también afirmativa, la pregunta definitiva es acerca 
de la existencia de crisis de reagudización del dolor a pesar de ese buen control basal y sólo si la respuesta es sí podemos diagnosticarle al paciente un dolor irruptivo; hemos localizado el pie que estuvo en el baile, usó ese zapato y lo perdió en su precipitada huída.

\section{TRATAMIENTO}

La clave del tratamiento del DIO son los opioides y toda la investigación y práctica clínica giran en torno a encontrar el fármaco ideal administrado por la mejor vía. Por supuesto, el control del dolor crónico -mediante el uso de opioides pero también de fármacos del primer escalón de la OMS y de coadyuvantes y/o coanalgésicos si son precisos-, es un paso previo ineludible a la hora de plantearse el manejo del DIO; dolor crónico y dolor irruptivo son diferentes pero relacionados y la analgesia de ambos es complementaria.

En La Cenicienta todo gira en torno al hipotético pie que encajará en la realidad del zapato perdido; ese pie es la solución al enigma y el principio de la dicha de su dueña y del príncipe que ha buscado por todo el reino hasta dar con ella pero el dichoso zapato no encaja en el pie de ninguna de las invitadas al baile de palacio a pesar de haberse recorrido todas las casas e intentado calzarlo a rubias y morenas, altas y bajas, guapas y feas.... a todas. Nada.

En la casa donde vive Cenicienta las hermanastras tampoco han podido engañar al emisario, a pesar de mutilarse una un dedo y otra el talón para ajustarse a la horma, en un intento desesperado de ajustar el contenido al continente, como un lecho de Procusto horriblemente voluntario. ¿No hay más chicas en la casa? pregunta el cortesano. No, responden, a excepción de la criada, pero es imposible que sea su zapato, viste de harapos y vive en la cocina. Aún así, replica el infatigable buscador, vamos a intentarlo. Se intenta y ¡oh, maravilla!, el pie se desliza y encaja perfectamente en el zapatito, pues la Cenicienta es su propietaria, la futura prometida del príncipe, la belleza oculta en las cenizas.

Para tratar el DIO también se han utilizado fármacos ${ }^{(15)}$ que de ninguna manera se ajustan "a la horma", por diversas razones: en primer lugar, por no haber otros mejores $y$, en segundo, por razones económicas y de costumbre. Pero, ante la petición desesperada ¿no hay otra forma de hacerlo? aparecen los fármacos de liberación inmediata basados en el fentanilo y aplicados por vía transmucosa....y el pie encaja en el zapato sin que sobre dedo por delante ni talón por detrás.

Los fármacos conocidos como LAO (opioides de acción prolongada) son los habitualmente usados para tratar el dolor crónico, pues su cómoda posología disminuye el número de administraciones haciendo posible darlos cada 12 o 24 horas para las formas orales (morfina, oxicodona, hidromorfona y tapentadol) o bien cada 72 o 96 horas (formas trasdérmicas de fentanilo y de buprenorfina). En lo que se refiere a los SAO (opioides de liberación rápida, que no inmediata) son adecuados para la titulación de la dosis idónea de opiode en el dolor crónico y para la prevención de episodios predecibles de DIO como son los incidentales de carácter volitivo y los procedimentales; sin embargo, se han usado y todavía se usan en el DIO idiopático e incidental no volitivo, cuya aparición es impredecible en el tiempo, por lo que únicamente puede tratarse y debe hacerse de la forma más precoz posible con los fármacos más rápidos disponibles ${ }^{(10)}$.

Los ROO (opioides de liberación inmediata) son los únicos opioides diseñados específicamente para el tratamiento del DIO, en virtud de las características habituales de sus episodios. Todos ellos se basan en el fentanilo aplicado por vía trasmucosa, el cual no es un recién llegado 
a escena pues se dispone del comprimido transmucosa oral con aplicador (ACTIQ $\left.{ }^{\circledR}\right)$ desde hace ya 10 años; a él se han sumado posteriormente el comprimido por vía sublingual (ABSTRAL $($ )), el comprimido efervescente de administración bucal y sublingual (EFFENTORA®) y las formas para aplicación nasal (PECFENT® e INSTANYL $($ ). Independientemente de sus distintas características farmacocinéticas, de su biodisponibilidad y de su toxicidad local, cualquiera de ellas permite un control del DIO superior al obtenido por los opioides de liberación rápida que se han usado tradicionalmente, con excepción del dolor incidental previsible, como es el incidental volitivo y el procedimental, pues para ambos puede ajustarse el tiempo de espera hasta el efecto analgésico adaptando el momento de la toma. Para el dolor incidental no volitivo y para el idiopático, una espera de 30 minutos hasta la analgesia y una duración del efecto de 4 horas suponen un desajuste cronológico importante.

Por lo tanto, igual que la danzarina misteriosa resultó revelada al demostrar que su pie se correspondía con el zapato, el tratamiento idóneo del DIO se manifiesta en los resultados de los estudios pivotales de los distintos fármacos ${ }^{(16-24)} y$, por supuesto, en la práctica clínica diaria, que es donde realmente se conoce lo que los diferentes fármacos ofrecen a los pacientes.

Respecto a las posibles condiciones para un buen uso de los ROO en el DIO, hay que volver al cuento, donde la elección de la prometida no parecía estar condicionada a ningún hecho físico o social pero esa impresión se produce porque las restricciones ya se habían establecido antes: las invitadas al baile de palacio eran todas ellas jóvenes pertenecientes a las mejores familias; de esa forma, el príncipe no rebajaba demasiado sus expectativas pues, sin duda, serían las mejor educadas, las más elegantes y vistosas, aptas para la vida palaciega. El que la vencedora fuera una falsa criada y una falsa fea no cambia para nada el hecho de que se buscaba entre la flor y la nata.

Respecto a las limitaciones establecidas para la protagonista, recordemos que el baile no terminaba a medianoche pero la condición para que a ella le fuera permitido acudir pasaba, irremediablemente, por este hecho: al sonar las doce campanadas había que volver a casa o desaparecería el hechizo y tanto el atuendo como la carroza se esfumarían.

Pues en el caso del uso de los ROO para el DIO, la condición normativa de uso es la titulación es decir, el ascenso gradual de la dosis desde la más baja existente para el producto, hasta lograr la dosis mínima eficaz, capaz de controlar el dolor sin importantes efectos adversos. La titulación es importante porque no se han establecido dosis equianalgésicas entre los opioides de vida media o larga y los de liberación inmediata indicados en el DIO y tampoco entre las diversas presentaciones de los fentanilos trasmucosos. En ausencia de estudios randomizados diseñados para comparar la dosificación por titulación frente a otras alternativas, la opción mayoritaria es titular ${ }^{(25)}$.

\section{CONCLUSIONES. FINAL FELIZ}

En los cuentos de hadas, se considera un final lo que, en realidad, es el principio de su parte más difícil: los enamorados deben conocerse y convivir; comer perdices es fácil si se vive en territorio de caza y se saben cocinar pero ser felices es otro cantar pues, aparte de la atracción física y la pasión amorosa, se precisa paciencia, empatía y muchas ganas de triunfar en el empeño; nuestros protagonistas son jóvenes, guapos y de buen corazón pero parecen saber poco de la vida y deben encajar la muy probable malcrianza principesca con la vida oscura en el entorno de un fogón. 
En la cuestión del dolor irruptivo en Oncología pasa algo parecido: sabemos distinguirlo, podemos tratarlo y disponemos de unos fármacos excelentes, de eficacia y seguridad probadas pero....hay que traspasar todo eso a la práctica clínica y preguntar en la consulta a los pacientes (empatía), titular las dosis hasta encontrar la óptima (paciencia) y prescribirlos con la convicción de que controlar el dolor es una parte fundamental de nuestra tarea médica (ganas de triunfar en el empeño).

Desconocemos si los protagonistas del cuento encontraron la dicha en el matrimonio, aunque pensamos que tenían muchas posibilidades de lograrlo, ya que lucharon por vencer dificultades que parecían insalvables. Nosotros, como médicos, lo que deseamos es un resultado satisfactorio para nuestros pacientes de la vida real, de modo que podamos decir:

$Y$ fueron felices... sin sufrimiento innecesario, sin privación de vida social y laboral, sin sensación de impotencia y desesperación... sin dolor.

\section{REFERENCIAS BIBLIOGRÁFICAS}

1. Grimm, Jacob \& Grimm, Wilhelm. Todos loscuentos de los hermanos Grimm. Ed. Rudolf Steiner \& Mandala. 2006.

2. Perrault Charles. La Cenicienta. Ed. Primera Copia, 2011.

3. Davies AN, Dickman A, Reid C, Stevens AM, Zeppetella G. The management of cancer-related breakthrough pain: Recommendations of a task group of the Science Committee of the Association for Palliative Medicine of Great Britain and Ireland. Eur J Pain 2009;3:331-8. Doi: 10.1016/j. ejpain.2008.06.014

4. Portenoy RK, Hagen NA. Breakthrough pain: Definition, prevalence and characteristics. Pain 1990;41:273-81.

5. Mercadante, S., Radbruch, L., Caraceni, A., Cherny, N., Kaasa, S., Nauck, F., Ripamonti, C., De Conno, F. and Steering
Committee of the European Association for Palliative Care (EAPC) Research Network. Episodic (breakthrough) pain. Cancer 2002; 94: 832-9. Doi: 10.1002/cncr.10249.

6. Portenoy R K, Forbes K, Lussier D, Hangs G. Difficult pain problems: An integrated approach. En: Doyle D, Hanks G, Cherny N, Calmen K, editores. Oxford Textbook of Palliative Medicine, 3a ed. Oxford University Press: Oxford; 2004.p.438-58.

7. Bruno Bettelheim. Psicoanálisis de los cuentos de hadas. Ed. Crítica. 2006.

8. Porta-Sales J, Garzón Rodriguez C, Juliá Torras J, Casals Merchán M. Dolor irruptivo em cáncer. Med Clin (Bar) 2010;135(6):280-5.

9. Greco MT, Corli O, Montanari M, Deandrea S, Zagonel V, Apolone et al. Epidemiology and pattern of care of breakthrough cancer pain in a longitudinal sample of cancer patients: Results from the Cancer Pain Outcome Research Study Group. Clin J Pain 2011,27(1):9-18. Doi: 10.1097/ AJP.0b013e3181edc250.

10. Bennett D, Burton AW, Fishman S, Fortner B, McCarberg B, Miaskowski C, et al. Consensus panel recommendations for the assessment and management of breakthrough pain. Part 2. Management P\&T 2005;30(6):354-60.

11. Mercadante S. Characteristics and mechanisms of BTCP. Eur J Palliat Care 2010;17 (3):8-12.

12. Zepetella G, Ribeiro MD. Pharmacotherapy of cancer-related episodic pain. Expert Opin Pharmacother 2003;4:493-502. Doi: 10.1517/eoph.4.4.493.22220

13. Hagen NA, Biondo P, Stiles C. Assessment and management of breakthrough pain in câncer patients: Current approaches and emerging research. Curr Pain Headache Rep 2008;12:241-8. Doi: 10.1007/ s11916-008-0042-1

14. Laverty DM, Davies A. Assessment. In: Davies A, editor. Cancer-related breakthrough pain. Oxford: Oxford University Press; 2006.p.23-30. 
15. Zeppetella G. Succesful management of breakthrough cancer pain. London. Evolving Medicine Ltd 2010.

16. Coluzzi P, Schwartzberg L, Conroy J Charapata S, Gay M, Busch MA, et al. Breakthrough cancer pain: a randomized trial comparing oral transmucosal fentanyl citrate (OTFC) and morphine sulfate immediate release (MSIR). Pain 2001;91:12330. Doi:1016/S0304-3959(00)00427-9

17. Farrar JT, Cleary J, Rauch R Busch M, Nordbrock $E$. Oral transmucosal fentanyl citrate: randomized, double-blinded, placebo-controlled trial for treatment of breakthrough pain in cancer patients. J Natl Cancer Inst 1998;90(8):611-6.

18. Portenoy RK, Taylor D, Messina J, Tremmel L. A randomized, placebocontrolled study of fentanyl buccal tablet for breakthrough pain in opioidtreated patients with cancer. Clin J Pain 2006;22:805-11.Doi:10.1097/01. ajp.0000210932.27945.4a

19. Slatkin NE, Xie F, Messina J, Segal TJ. Fentanyl buccal tablet for relief of breakthrough pain in opioid-tolerant patients with cancer-related chronic pain. J Support Oncol 2007;5(7):327-34.

20. Rauck R, Reynolds L, Geach J, Bull J, Stearns L, Scherlis M, Parikh N, Dillaha L. Efficacy and safety of fentanyl sublingual spray for the treatment of breakthrough cancer pain: A randomized, doubleblind, placebo-controlled study. Curr Med Res Opin. 2012;28(5):859-70. Doi: 10.1185/03007995.2012.683111

21. Portenoy RP, Burton AW, Gabrail N, Taylor D. Fentanyl Pectin Nasal Spray 043 Study Group. On behalf of the Fentanyl
Pectin Nasal Spray 043 Study Group. A multicenter placebo-controlled, doubleblind, multiple-crossover study of fentanyl pectin nasal spray (FPNS) in the treatment of breakthrough cancer pain. Pain 2010;151(3):617-24. Doi: 10.1016/j. pain.

22. Davies A, Sitte T,Elsner F, Reale C, Espinosa J, Brooks D, Fallon M. Consistency of efficacy, patient acceptability, and nasal tolerability of fentanyl pectin nasal spray compared with immediate-release morphine sulfate in breakthrough cancer pain. J Pain Symptom Manage 2011; 41(2):358-66. Doi: 10.1016/j.jpainsymman.2010.11.004

23. Kress HG, Ororiska A, Kaczmarek Z, Kaasa S, Colberg T, Nolte T. Efficacy and tolerability of intranasal spray 50 to 200 mg for breakthrough pain in patients with cancer: a phase III, multinational, randomized, doubleblind, placebo-controlled, crossover trial with a 10-month, open-label extension treatment period. Clin Ther 2009; 31:1177-91. Doi: 10.1016/j.clinthera.2009.05.022

24. Portenoy RK, Raffaeli W, Torres L Sitte T, Deka AC, Herrera IG et al. Long-term safety, tolerability, and consistency of effect of fentanyl pectin nasal spray for breakthrough cancer pain in opioid-tolerant patients. J Opioid Manag 2010;6(5):319-28.

25. Hagen NA, Fisher K, Victorino Victorino C Farrar JT. A titration strategy is needed to manage breakthrough cancer pain effectively: Observations from data pooled from three clinical trials. J Palliat Med 2007; 10(1):47-55. Doi: 10.1089/ jpm.2006.0151. 